\title{
SandBOX - An Intuitive Conceptual Design System
}

\author{
Stamatios Psarras, Marcin Kosicki, Khaled El-Ashry, Sherif Tarabishy, \\ Martha Tsigkari, Adam Davis and Francis Aish \\ Applied Research + Development, Foster + Partners
}

\begin{abstract}
We describe the development and use of a new conceptual design system, called SandBOX, which combines a range of intuitive interfaces with real-time analysis, thus enabling a wide variety of users to develop performative concept designs. We show how this interactive design platform can overcome some of the limitations of current physical model-based design processes, whilst retaining many of their advantages.
\end{abstract}

Keywords: Performance Driven Design, Virtual Reality, Augmented Reality, Interoperability, Optimisation, Mixed Reality Design

\section{Challenge: Moving from the Physical to the Digital}

Tools for conceptual design have a number of challenging requirements. They must be very intuitive to allow users of all technical abilities to rapidly capture and express their ideas. They must also allow multiple users to evaluate and refine designs and permit effective collaboration. More importantly, they need to be able to allow for quick feedback loops regarding a variety of criteria in order to produce performance driven design solutions.
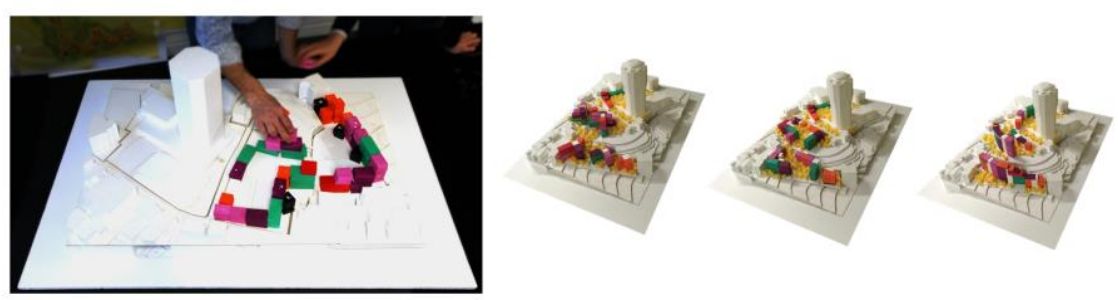

Fig. 1. Conventional conceptual modelling using foam blocks 
Analogue physical tools such as sketching and physical models have traditionally dominated the conceptual modelling space. An example of common practice is shown in Fig. 1, where physical foam blocks are prepared by cutting them out in small representative sizes and spray-painting them with different colours to represent different typologies or uses. Designers with different seniority and expertise can rapidly and intuitively gather around the physical model and experiment with different spatial arrangements or communicate their choices to a client.

Although this process is approachable, intuitive and clear, it has several significant limitations. First, there is a substantial economic and time cost in preparing the model. The right size of each block needs to be pre-determined and a certain number of blocks will have to be cut and painted. Any subsequent changes to programme, types or sizes lead to the process being repeated, resulting to either delays or avoidance of any changes, thus limiting the creative process. Secondly, such approaches are usually limited to one configuration at a time. Thirdly, after creating a possible option using a physical model, the resulting spatial configuration needs to be documented before starting another one. This is usually performed by photographing the physical model from different angles and then digitizing it in a 3D model. However, recording design options this way is often inaccurate and the process of digitization can be laborious. Moreover, some of the resulting design options would have to be communicated to consultants for further processing and analysis

Having to undergo all the above processes, the design feedback cycle can stretch to several days, by which point the design may have moved on considerably and the feedback from the consultants becomes irrelevant. The response time of analytical tools has been highlighted as a crucial aspect of performance-driven design in the early conceptual stages of design (Chronis et al. 2012). Another restriction is that interaction is effectively limited to participants co-located with the model; this can exclude remote collaborators, a common occurrence in design processes nowadays.

Conversely conventional digital tools such as CAD or BIM software have more flexibility in both representation and manipulation and can be directly linked to analysis tools. However, their mouse-based interfaces limit them to single user input, and 2D screens cannot provide a full experience of the design. Furthermore, many designers and clients find these interfaces un-intuitive and too complex to learn, which can lead to a disconnect between designer and representation, and the need for intermediaries to manipulate representations on their behalf.

\section{Prior art}

Research has focused on various advancements, such as novel simulation algorithms, interoperability frameworks and human computer interaction developments (Malkawi 2004). Despite the aforementioned, physical models are still frequently used, particularly during the concept stage (Sun et al. 2014) and often hybrid methods that mix the physical with the digital are used to help bridge the gap (Winder and Ma, 2017). At 
the same time, there is a growing need and appetite for computational, data driven approaches in design which necessitates digital analysis (Bier and Knight 2014). But attempts to transition from early physical models to new digital interfaces that can be integrated with subsequent digital tools, is often met with significant resistance (Doyle and Senske, 2017). This resistance to new non-physical interfaces may occur due to a) their steep learning curve b) their often-limited features, and finally c) their lack of engagement during presentations from non-designers.

Various forms of virtual modelling and design review systems combing real-time rendering, interactivity, collaboration, dynamic lighting and collision detection have been proposed and developed over the past 20 years, utilizing technologies such as Head-Mounted Displays (HMDs) (Fatah et al., 2005), 3D game engines (Shiratuddin and Thabet, 2011) and projection domes (Dorta et al., 2014).

The authors have previously developed and evaluated an array of collaborative performance-driven design applications, to assess how innovative interfaces can bridge the gap between physical and digital design and their evaluation thereafter. These included:

- A see-through AR HMD system with hand tracking and 3D input devices, with a real-time connection to a CAD system

- Touch-screen user interfaces that allowed for real time geometry generation and analysis.

- An AR system that used cameras and project mapping to capture and augment a physical model, where changes made to the physical model were synchronised with a digital model, analysed in real-time, and then projected back on the physical model.
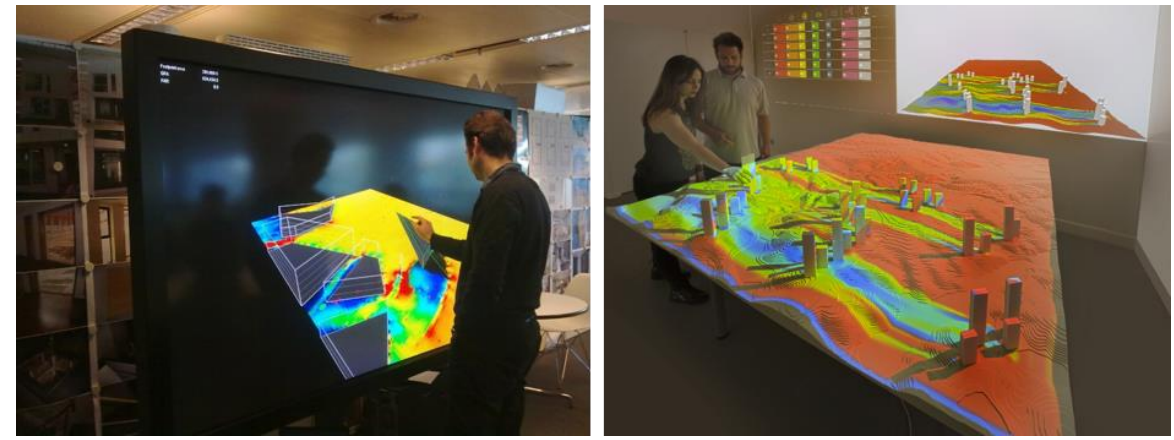

Fig. 2. Previous developments of interactive tools

All of the above were developed as custom applications, with import and export capabilities, as well as powerful built-in analysis engines, that worked real-time and 
evaluated design performance in terms of daylight potential, insolation, airflow, spatial and visual connectivity, views and pedestrian flow.

Most were successfully used on live projects, at a range of scales from floorplate layout to master-planning. Nevertheless, they had a number of limitations which constrained their use. Firstly, some of the hardware used was extremely expensive, or limited to a fixed location, which made it difficult to deploy widely. Secondly, the modelling capabilities only allowed for simple representations, making it difficult to design undercuts or work with occluded elements. Thirdly, the setup time for a lot of these applications was significant (placement of cameras, calibration time etc).

\section{SandBOX: An Intuitive Conceptual Design System}

SandBOX is the latest generation of interactive design tools developed by the authors, who are members of the Applied Research + Development group at Foster + Partners. Its development had a number of design goals: (i) to extend the knowledge gained from previous systems, and address some of their limitations. (ii) to take advantage of recently developed hardware and software, namely powerful and affordable AR/VR displays and games engines (iii) to be easy to set up and use in practice by designers (iv) to be useful at all scales of concept design, from interiors and floorplates, through buildings, to masterplans.

We describe four main aspects of the system: interfaces, modelling, analysis and interoperability. We also describe early use and evaluation of the system in commercial practice, and potential next steps. 


\subsection{Interfaces}
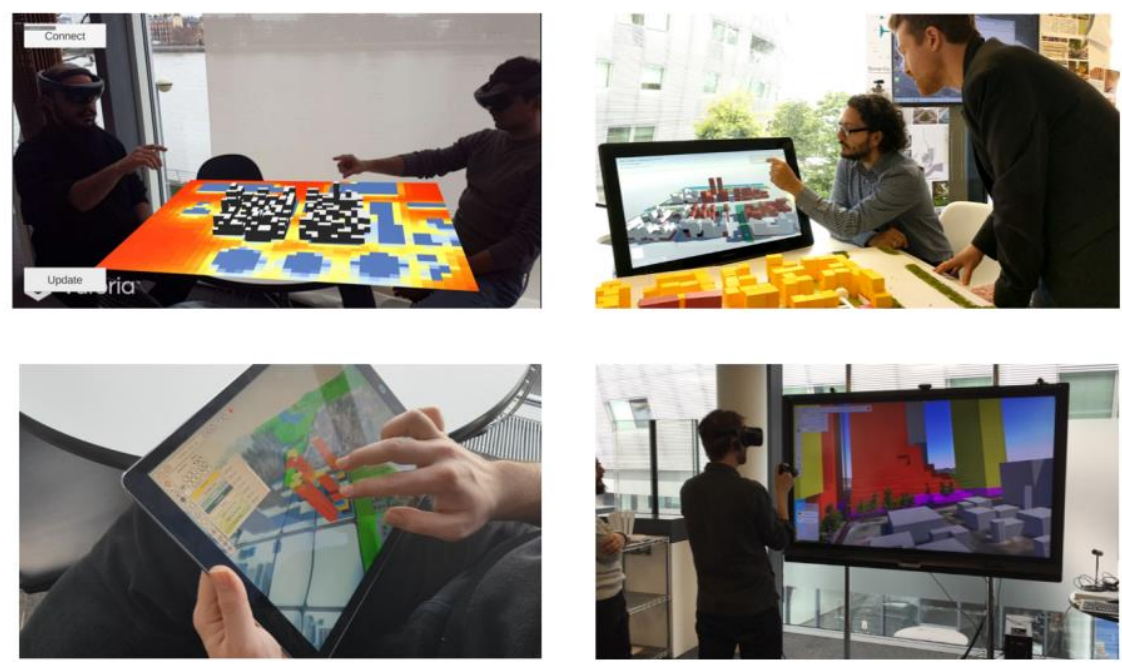

Fig. 3. Range of intuitive interfaces

Due to the wide range of potential users, it was important to provide a set of interfaces that allowed each user to find the ones they felt most creative in (see Fig 3). To that end, a number of interfaces were developed, integrated and evaluated, including virtual and augmented reality HMDs with hand tracking and controllers, large touch screens, tablets with touch and stylus inputs, as well as regular desktop computers. Most of these interfaces permit multi-user interaction, a key part of the conceptual design process. They have been evaluated with a wide range of users, both technical and non-technical, with most becoming familiar with the system in a matter of minutes, and some extremely fluent in hours.

The implementation of HMDs was especially successful due to their immersive capabilities. Visualization of architectural models is a subdomain of a wider problem of data visualization. Research carried on by Donalek et al, (2014) demonstrated that immersive VR could be effectively used for general purpose data exploration. Olshannikova et al (2015) identified filtering, scaling and combination of different visualization techniques as important interactive features used in contemporary data visualization. Of the aforementioned, scaling is the most important to architects because by using physical models at different scales, designers have attempted to experience architectural space in different scales simultaneously, and by employing various drawing techniques, to achieve an embodied perception of the designed space (Yaneva, 2005). SandBOX combined those features with three basic functions found in any CAD system, namely: pan, orbit and scale (zoom) and converted them into intuitive gestures specific for HMDs. Using the controllers, users can scale themselves by grabbing a digital model and pushing or pulling their hands apart or together (like 
zooming in and out). Additionally, they could grab the model and position it anywhere within their grasp (like panning). Finally, the controllers could be activated and turned like the steering wheel of a car to rotate the model around the up vector. These techniques proved highly intuitive and effective and had the added benefit of almost completely avoiding user motion sickness, which can be a common occurrence with other VR navigation techniques.
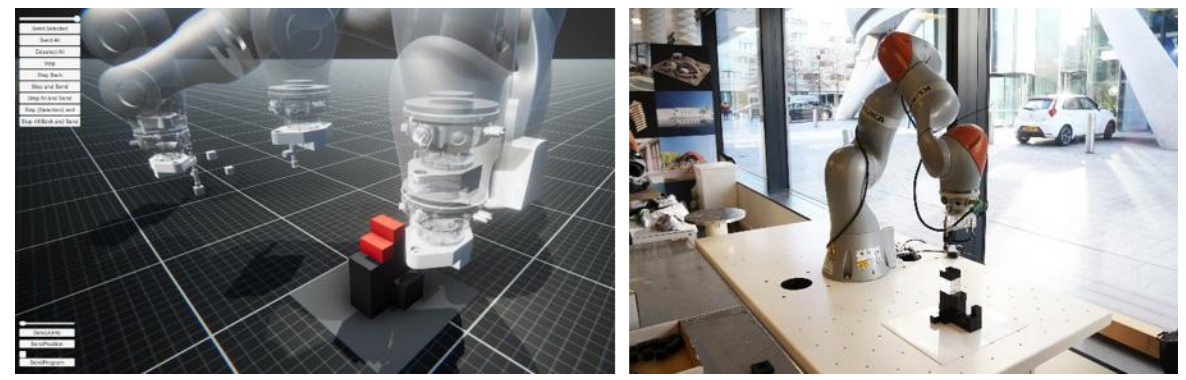

Fig. 4. Communication between the digital and the physical

An initial investigation was conducted into using a physical model representation as an output to our voxel model system using an automated pick and place method, which would in real time recreate a physical model from its digital representation (Fig 4). This was achieved by implementing an IK solver and path planner connected to an industrial robot with a pneumatic suction end effector. The pick and place operations were sent in real-time to the robot while the digital voxel model was being edited. However, this initial implementation is currently uni-directional, in future work we plan the implementation of a bi-directional system using machine vision to allow the physical model to act as input to the digital one.

\subsection{Modelling}

The system supports a range of geometric representations and modelling capabilities, shown in Fig. 5. The primary representation is that of a voxel, which is a direct analogy of the foam blocks used in physical models. The voxels can have a literal block representation or can be instances of more complex geometries. This flexibility allows the voxels to be used in a variety of different designs and scenarios. Users could 3D paint with voxels using different sized 'brushes', and also use a 'laser' to add and remove individual voxels at a distance. The system also supports placement of instances of existing 3D models, such as trees. 

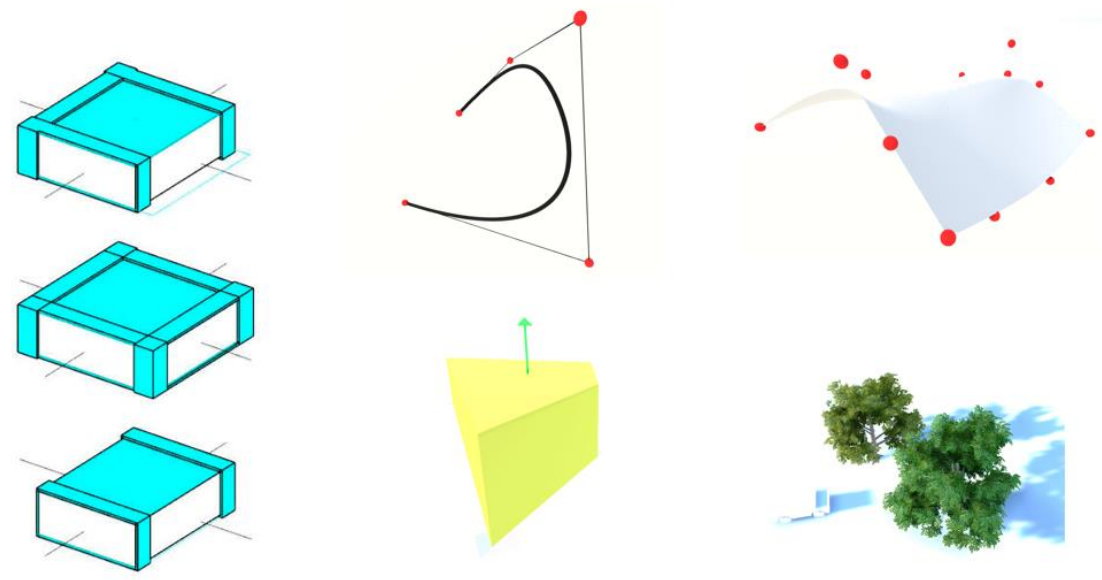

Fig. 5. Range of geometric representations

The system also allows placement and manipulation of b-spline curves and surfaces, both for more free-form expression, but also (in the case of the former) for annotating and commenting purposes. Finally, a polygonal mesh sculpting module was implemented (see Fig. 6), with a particular focus on terrain modelling. Controls include the ability to sculpt and smooth the mesh in a clay-like fashion or snapping to specific heights. Feedback is given by changing the representation of the mesh to indicate the height gradient and the contours.

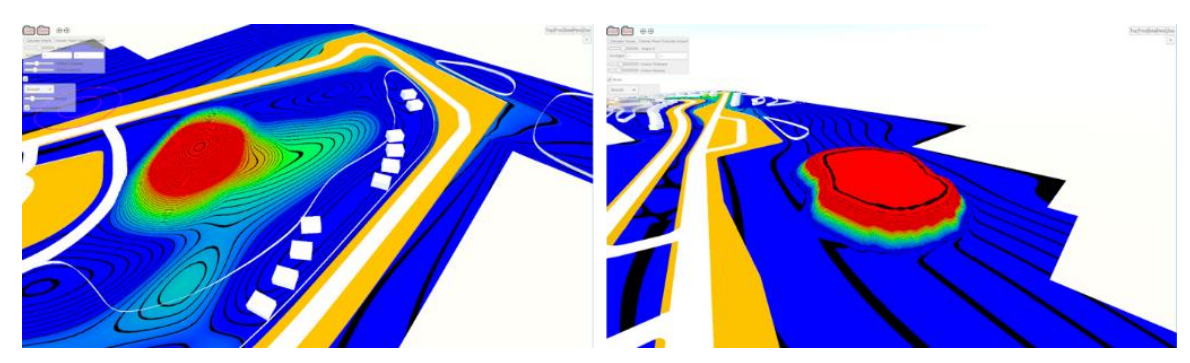

Fig. 6. Sculpting tools

The wide range of available tools was particularly successful in accommodating different scenarios that users wanted to explore at early design stages. The ability to instantly create and recall design options was another critical advantage over physical models. 


\subsection{Analysis Tools and Metrics}

The primary objective for integrating preliminary performance analysis into the system was to empower users with real or near-real-time feedback on their designs. This allowed users to seamlessly move from modelling to analysing and modifying the geometry accordingly in real time. This reduced the design cycle time and allowed users to spend more time on being creative.

SandBOX was designed to integrate a range of in-house analysis engines. Specific examples include Quality of View to assess the views from the façade and Vertical Sky Component (VSC) to assess the daylight potential of the building. Since we adopted a voxel-based approach it was natural to also include graph metrics based on Space Syntax analysis namely, spatial and visual connectivity (Turner et al., 2001) as well as pedestrian simulations (Turner and Penn, 2002). One key challenge with physical models is accurately keeping track of quantity metrics, e.g. how many blocks of a certain typology have been placed. This is done automatically in SandBOX, with live check against the project brief. Whilst very simple, this was a massive time saver and confidence giver for the teams using the system. As well as basic brief checks, the system could also be linked in real-time to external financial models, to assess the financial viability of different options. This analysis and other more sophisticated simulations were linked using a custom data exchange system described in the next section. To maintain a smooth user experience, all analysis engines were nonblocking on the user interaction loop, and additional algorithmic refinements led to performance increases compared to previous systems, up to 400x $\mathrm{n}$ the case of spatial connectivity.
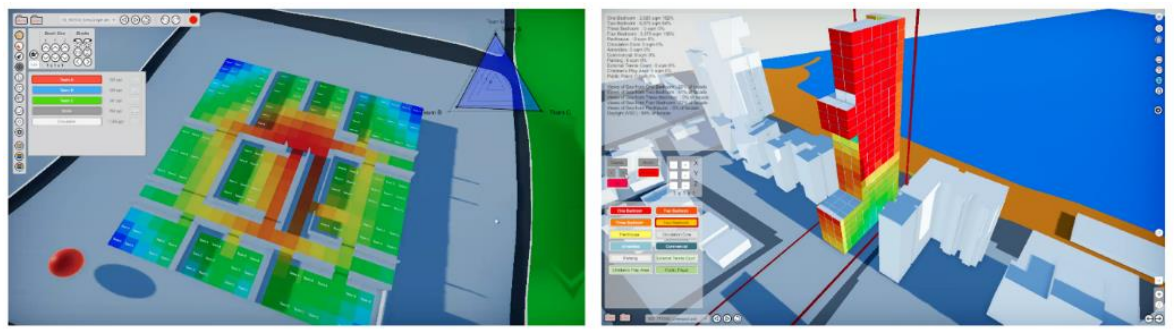

Fig. 7. Example of different analysis results. Left connectivity of a floorplate, Right vertical sky component on the facade of a tower 


\subsection{Interoperability}

Interoperability issues have been identified as one of the key obstacles to seamless collaboration between various component of design systems (Flager and Haymaker, 2007) (Muller et al., 2013). Therefore, the ability to create bi-directional connections to multiple CAD, BIM and simulations environments was a major implementation goal. This was achieved using two techniques. Firstly, a custom file format was developed, capable of incorporating all data required to capture a given design state These files could be set up in parametric systems such as Grasshopper, and defined context models, design briefs, and which modelling or analysis modules to enable. This avoided non-technical end-users having to do any complex setup, as all configuration was in one file. Then, massing models created using the system could be exported using the same file format and automatically recreated in CAD and BIM systems such as Rhinoceros or Revit, using platform-specific native geometry ensuring seamless integration with the main design pipeline. Secondly, the system integrated a custom real-time data exchange system called Hermes, also developed by the authors. This allowed real-time bi-directional exchange of geometry and analysis to a wide range of other applications. This was particularly useful for other analysis engines which could not be directly integrated into the system for technical or commercial reasons.

\section{$4 \quad$ Reception and Feedback}

The development of SandBOX was performed in parallel and in conjunction with a number of active design projects. This resulted in a close collaboration between the development team and the design teams using the application, with feedback from the design teams was incorporated throughout the process. This collaborative approach ensured that SandBOX met the needs of its intended users and accelerated its dissemination in the practice. SandBOX has been used on over 30 live projects in the past 2 years, ranging from masterplans, to buildings and floorplate layouts. It has been evaluated by an extensive internal user community, from very senior designers to 'digital natives', as well as by external clients

To assess the impact of SandBOX on the design pipeline we interviewed multiple experienced architects in our practice regarding the time it takes to model, archive, analyse and compare different design options. We found that implementation of SandBOX reduced the total time spent by a factor of 8 (Fig 8). 


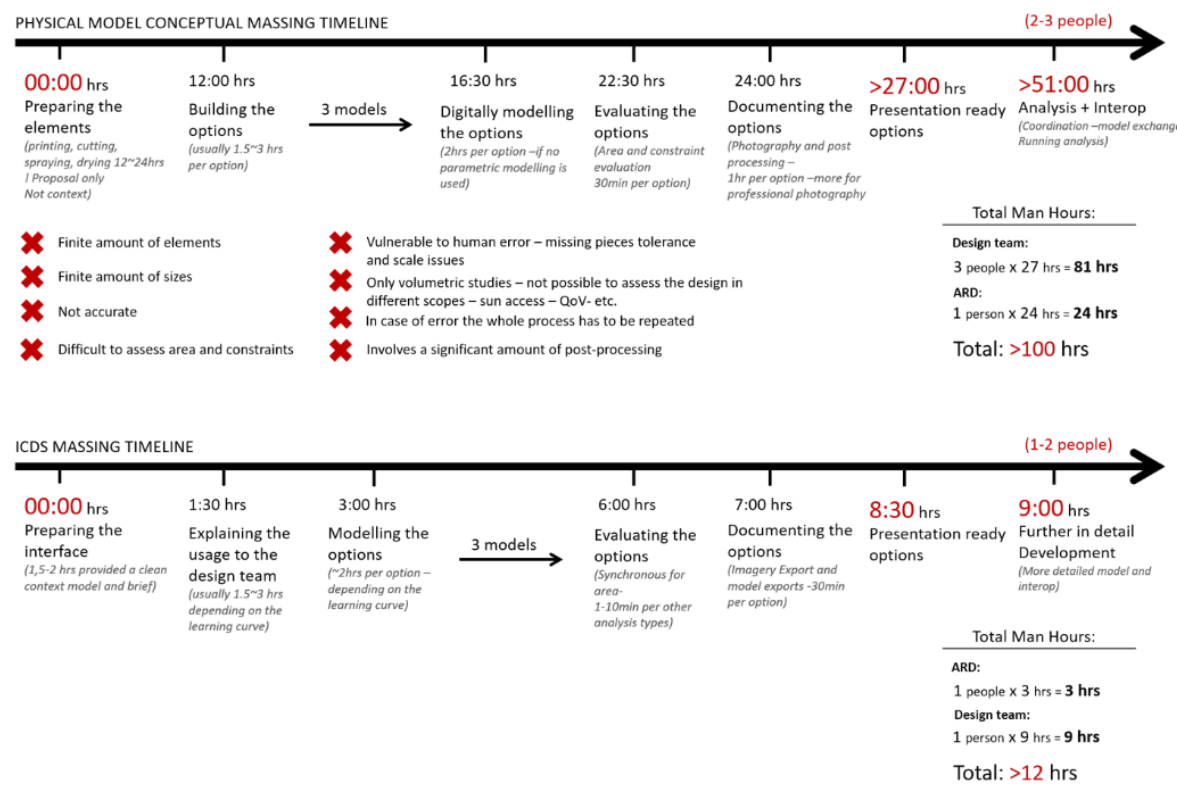

Fig. 8. Process steps and time spent when using a physical model (Top), compared to when using SandBOX. We estimate that a team can achieve 8x performance increase. This further increases with the number of models that are produced and with the familiarity the team has with the tool.

SandBOX has taken the role for delivering massing of buildings quickly and easily. Since it has been based on an intuitive physical-inspired process that was used extensively within our practice, transitioning to a digital equivalent was easier. The system has been used by senior designers as a means of communicating their ideas to the rest of the team; this is especially true for the VR interface where the controls act much like a pencil when sketching. Communicating in this way to junior members of the team can convert a rough idea into a much more specific design, avoiding the hurdles of decoding and digitising a crude sketch. Feedback from design teams suggested that the interoperability capabilities of the system enable a more integrated digital process, which reduces unnecessary remodelling and interpretation, and allows more time to the team to develop and refine the design. The VR version was also informally evaluated at a public exhibition, with more than 2000 visitors over the course of two days. Through this experience we gathered comments from users with and without architectural backgrounds, of wide-ranging ages and without prior experience of the system. We were broadly commended over the intuitiveness of the system. We also observed extremely few users reporting any motion sickness or discomfort, a problem which is far more common with other VR systems that use a less intuitive navigation technique. 


\section{$5 \quad$ Next steps}

Initial user feedback has been good, but we intend to continue developing and improving this system. Potential for future work include:

- Enhancing the modelling tools with more capabilities, such as solid modelling, and more sophisticated manipulation tools.

- Incorporating more analyses into the system, and take more advantage of both local and remote compute resources.

- Investigating new AR hardware to bring the virtual closer to the physical.

- Enhancing the physical - digital collaboration, where editing blocks on a physical model updates the digital voxel model. This would allow designers surrounding the physical model to have the freedom to manually edit it while working with other remote users using the digital application.

\section{Acknowledgements}

We would like to thank our past and current colleagues for their contributions to the development and evaluation of the system, in particular Vassilis Papalexopoulos and David Gillespie.

\section{References}

1. Bier, Henriette, and Knight, Terry. 2014. "Data-Driven Design to Production and Operation." FOOTPRINT, November, 1-8. https://doi.org/10.7480/footprint.8.2.807.

2. Chronis, A., M. Tsigkari, A. Davis, and F. Aish. 2012. "Design Systems, Ecology, and Time" shttps://core.ac.uk/display/15359344.

3. Donalek, Ciro, S. G. Djorgovski, Scott Davidoff, Alex Cioc, Anwell Wang, Giuseppe Longo, Jeffrey S. Norris, et al. 2014. "Immersive and Collaborative Data Visualization Using Virtual Reality Platforms.” 2014 IEEE International Conference on Big Data (Big Data), October, 609-14. https://doi.org/10.1109/BigData.2014.7004282.

4. Doyle, Shelby, and Nicholas Senske. n.d. "Between Design and Digital: Bridging the Gaps in Architectural Education." Accessed April 12, 2019. https://www.academia.edu/25663417/Between_Design_and_Digital_Bridging_the_Gaps_i n_Architectural_Education.

5. Kinayoglu, Gokce, and Tomás Dorta. n.d. "Hyve-3D: A New Embodied Interface for Immersive Collaborative 3D Sketching." Accessed April 11, 2019. https://www.academia.edu/8241641/Hyve3D_A_New_Embodied_Interface_for_Immersive_Collaborative_3D_Sketching. 
6. Olshannikova, Ekaterina, Aleksandr Ometov, Yevgeni Koucheryavy, and Thomas Olsson. 2015. "Visualizing Big Data with Augmented and Virtual Reality: Challenges and Research Agenda." Journal of Big Data 2 (1): 22. https://doi.org/10.1186/s40537-015-00312.

7. Schieck, Ava Fatah gen, Alan Penn, Chiron Mottram, Andreas Strothmann, Jan Ohlenburg, Wolfgang Broll, Francis Aish, and Simon Attfield. 2005. "Interactive Space Generation Through Play Exploring the Role of Simulation on the Design Table." International Journal of Architectural Computing 3 (1): 3-25. https://doi.org/10.1260/1478077053739612.

8. Shiratuddin, Mohd Fairuz, and Walid Thabet. 2011. "Utilizing a 3D Game Engine to Develop a Virtual Design Review System." Journal of Information Technology in Construction (ITcon) 16 (4): 39-68. http://www.itcon.org/paper/2011/4.

9. Sun, Lei, Tomohiro Fukuda, Toshiki Tokuhara, and Nobuyoshi Yabuki. 2014. "Differences in Spatial Understanding Between Physical and Virtual Models." Frontiers of Architectural Research 3 (1): 28-35. https://doi.org/10.1016/j.foar.2013.11.005.

10. Turner, Alasdair, Maria Doxa, David O'Sullivan, and Alan Penn. 2001. "From Isovists to Visibility Graphs: A Methodology for the Analysis of Architectural Space.” Environment and Planning B: Planning and Design 28 (1): 103-21. https://doi.org/10.1068/b2684.

11. Turner, Alasdair, and Alan Penn. 2002. "Encoding Natural Movement as an Agent Based System: An Investigation into Human Pedestrian Behaviour in the Built Environment." Environment and Planning B: Planning and Design 29 (4): 473-90. https://doi.org/10.1068/b12850.

12. Winder, James Ira, and Cambridge Ma. 2017. "Tangible Interactive Matrix for Real-Time Computation and 3D Projection Mapping," 5.

13. Yaneva, Albena. 2005. "Scaling up and down: Extraction Trials in Architectural Design." Social Studies of Science - SOC STUD SCI 35 (December): 867-94. https://doi.org/10.1177/0306312705053053. 